\title{
La separación funcional de las actividades monopólicas del mercado elécrrico chileno: una tarea pendiente*
}

\author{
Recibido: 16 de noviembre de 2018 • Aprobado: 13 de mayo de 2019 \\ https://doi.org/10.22395/ojum.v18n37a2 \\ Óscar Guillermo Guzmán Zepeda*
}

\begin{abstract}
RESUMEN
El artículo busca explicar, a partir de la regulación económica, los riesgos que plantea la existencia de empresas verticalmente integradas en el sector eléctrico y la importancia de la separación de actividades. De esta forma, a partir de las diversas técnicas regulatorias que ofrecen, tanto la doctrina como los modelos comparados, particularmente de la Unión Europea y España, se recomienda avanzar en el sector eléctrico chileno hacia una separación funcional de actividades.

Palabras clave: integración vertical; sector eléctrico; regulación económica; separación de actividades; separación funcional.
\end{abstract}

El presente artículo proviene de la actividad investigativa del autor en el área de la regulación económica del sector eléctrico, en el marco de sus investigaciones doctorales.

** Licenciado en Ciencias Jurídicas, Universidad de Talca, Talca, Chile, candidato a Doctor en Derecho, Universidad de Talca, Talca, Chile. Profesor instructor de la Escuela de Derecho de la Universidad Santo Tomás sede Talca, Chile. Correo electrónico: oscarguzmanzepeda@gmail.com. Orcid: https://orcid. org/0000-0001-7615-1508 


\title{
The Functional Separation of Monopolistic Activities in the Chilean Electricity Market: a Pending Task
}

\section{ABSTRACT}

This article pretends to explain, from economic regulation, the risks of vertically integrated companies in the electricity market and the importance of the separation of activities. Parting from diverse regulatory techniques offering, both from doctrine and comparative models, particularly in the European Union and Spain, and advancement in the Chilean electricity sector is recommended towards a functional separation of activities.

Keywords: vertical integration; electricity sector; economic regulation; activities separation; functional separation.

\section{A separação funcional das atividades monopólicas do mercado elétrico chileno: uma tarefa pendente}

\begin{abstract}
RESUMO
Este artigo pretende explicar, a partir da regulamentação econômica, os riscos que a existência de empresas verticalmente integradas apresenta no setor elétrico e a importância da separação de atividades. Dessa forma, a partir das diversas técnicas de regulamentação oferecidas, tanto a doutrina quanto os modelos comparados, particularmente da União Europeia e da Espanha, recomenda-se avançar no setor elétrico chileno a uma separação funcional de atividades.

Palavras-chave: integração vertical; setor eléctrico; regulamentação econômica; separação de atividades; separação funcional.
\end{abstract}




\section{INTRODUCCIÓN}

El modelo regulatorio del sector eléctrico, en un sistema liberalizado como el chileno, se confía en la iniciativa privada, la libertad y la competencia para lograr crear un mercado de la electricidad que brinde un servicio de calidad continuo, regular y a precios accesibles para los consumidores. Para estos efectos, la regulación tiene una misión esencial: crear las condiciones que permitan un mercado competitivo, dentro de lo cual es menester asegurar una real y efectiva separación de actividades, desintegrando verticalmente aquellos segmentos que se presenten como monopolio natural de aquellos que no lo son.

La separación de actividades dentro del mercado eléctrico busca establecer barreras entre segmentos que se presentan de manera monopólica, como es el transporte o transmisión; de otros que pueden desarrollarse en un régimen de competencia, como son la generación, o en algunas legislaciones, la comercialización. El peligro que encierra controlar estas actividades en una misma empresa, grupo o persona radica en que estas gozarían de un poder de mercado por sobre los demás actores, perjudicando una real competencia en el sector.

En el caso chileno, la Ley General de Servicios Eléctricos -en adelante LGSE- (Decreto con Fuerza de Ley n. ${ }^{\circ}$ 4/20.018 de 2006) en el artículo 7 impone ciertos requisitos y deberes para las sociedades que se dedican al transporte de electricidad por el sistema de transmisión, buscando que estas no participen en actividades de generación y distribución, como también limita la participación en el sistema de transmisión de empresas dedicadas a otros rubros del sistema eléctrico. Ante este escenario que presenta nuestra regulación, cabe preguntarse si dichas medidas significan -o nouna real separación de actividades, o si solo es una separación parcial y, de ser así, cómo se puede avanzar para lograr dicho objetivo.

En atención al interrogante antes planteado, se ofrece como hipótesis que la actual regulación económica del mercado eléctrico solo asegura una separación de actividades jurídica y contable, pero carece de mecanismos e instituciones que aseguren una separación funcional entre los operadores de las líneas de transporte con los demás actores del mercado. Así las cosas, el objetivo general del presente trabajo es identificar las deficiencias del esquema de separación de actividades en el mercado eléctrico, para así proponer modificaciones regulatorias que permitan avanzar hacia una real autonomía y separación en el sector.

Respecto a la metodología empleada, al ser esta investigación principalmente orientada al campo de las ciencias jurídicas, se utilizó el método dogmático, de forma que se procedió a recopilar, seleccionar, sistematizar y analizar críticamente las fuentes 
doctrinarias -nacionales y extranjeras-y normativas sobre la temática. De igual forma, se recurrió al método comparativo, debido a que las materias objeto de esta investigación han sido analizadas extensamente en ordenamientos jurídicos extranjeros.

En cuanto a la estructura del artículo, en primer lugar, se explica el papel que debe cumplir la regulación económica en los procesos de liberalización del sector eléctrico, luego se analiza la separación de actividades y desintegración vertical de los mercados, tanto en el modelo nacional como también en regulaciones extranjeras, para así poder identificar las deficiencias del sistema y concluir con propuestas destinadas a avanzar en la materia.

\section{LA REGULACIÓN ECONÓMICA DEL MERCADO ELÉCTRICO EN LOS PROCESOS DE LIBERALIZACIÓN}

Dar un concepto unívoco de lo que es regulación económica no resulta sencillo, por cuanto puede ser visto desde distintas disciplinas, como la economía, la politología, la sociología o el derecho. Sumado a lo anterior, la incertidumbre es mayor a partir de los procesos de liberalización y privatización que crean en los ordenamientos jurídicos nuevos y novedosos mecanismo de intervención estatal en la actividad económica liberalizada!'.

Por otro lado, el desarrollo del concepto en el derecho continental y en el derecho anglosajón tiene importantes diferencias, ya que mientras en el primero se tiende a catalogar la intervención pública en la actividad social y económica a través de las distintas formas de actuación estatal, en el segundo se le da un sentido más restringido, como una intervención en la actividad económica por parte de agencias de la administración (Muñoz, 2009, p. 16; Betancor, 2010, pp. 31-33). De igual forma, en atención a la finalidad perseguida por medio de la regulación, se pueden apreciar dos maneras de entender el concepto: una amplia y otra restringida. Respecto a la primera, la regulación agrupa las diversas formas de actuación mediante instrumentos jurídicos ensayadas por los actores estatales con el objeto de satisfacer intereses generales, particularmente en actividades económicas (Darnaculleta, 2009, pp. 372-375).

Por su parte, la regulación -en un sentido restringido- hace referencia a una intervención o control continuo y concertado por las autoridades públicas del mercado mediante la imposición de obligaciones jurídicas a los particulares, con el fin de garantizar la adecuación del funcionamiento del mercado a los objetivos de interés general, en particular, intervenir un comportamiento comprendido dentro de la libre competencia (Montero, 2013, p. 86).

Este concepto y un análisis sobre el carácter pluridisciplinar de la regulación se encuentra en Betancor (2010, pp. 31-47). 
En atención a esta distinción, no cualquier intervención estatal en la economía puede ser calificada como regulación económica propiamente, pues pueden existir infinidad de normas con injerencia en la actividad económica, pero que no están dirigidas a modificar un comportamiento comprendido dentro de la libre competencia. De este modo, las normas de protección al consumidor, de protección al medioambiente, de estándares sanitarios, régimen tributario, etc., si bien intervienen la actividad económica y pueden ser consideradas de regulación en un sentido amplio, no necesariamente serán regulación económica (Sepúlveda, 2010, p. 11).

Esta influencia estatal en la conducta de las empresas que operan en el mercado a través de la fijación de deberes y prohibiciones solo puede estar justificada por tratarse de sectores en donde se intercambian servicios económicos de interés general (Millán, 2013, p. 115). Esta intervención se hará mediante diversos instrumentos, como son establecer requisitos de entrada, monopolios, fijación de precios y tarifas, regulación de las condiciones de actuación de los operadores, imposición de obligación de prestación del servicio universal, separación de actividades, entre otras (Darnaculleta, 2009, pp. 369-374)².

Esta intervención en la economía puede ser de una mayor o menor entidad dependiendo del ordenamiento jurídico de que se trate, de acuerdo con los principios que inspiren la distribución de funciones público-privadas en dicha sociedad. En el caso chileno, dicha distribución se fundamenta en la concepción que se adopta del principio de subsidiaridad ${ }^{3}$.

Para el profesor Bascuñán (1997), el principio de subsidiaridad tiene dos implicancias: por un lado, que el Estado no tome a su cargo lo que en buenas condiciones puedan realizar las personas o entes colectivos y, por otra parte, la obligación del Estado de satisfacer las necesidades colectivas cuando los particulares no estén en la posibilidad de lograrlo (p. 52). De esta manera, se busca restringir la actividad del Estado, de forma tal que solo podrá actuar cumpliendo ciertos requisitos, tales como que la respectiva actividad sea conveniente para el bien común, que los particulares no logren un nivel adecuado acorde a las exigencias de este bien común o no realicen

2 Explica la autora que la concepción estricta de la regulación se diferencia de la concepción amplia en que esta última comprende toda la actividad del Estado que tiene por objeto la prosecución, en el caso concreto, del bien común o de los intereses generales, teniendo en cuenta todos los instrumentos jurídicos de influencia estatal en los procesos sociales.

3 Siguiendo al profesor Frosini (2002, p. 8), este principio se puede entender en dos sentidos: vertical y horizontal. El primero hace referencia a la relación entre un ordenamiento supranacional (como la Unión Europea) con los ordenamientos nacionales o bien, la relación entre el Estado y los entes sometidos a él, pero dotados de autonomía, como las regiones, provincias o municipios. Por su parte, en su sentido horizontal, la subsidiaridad alude a la relación entre el Estado y los ciudadanos, tanto como individuos u entes intermedios, con el explícito propósito de dejar el mayor espacio de autonomía privada, reduciendo la intervención pública. 
dicha actividad, y finalmente que el Estado haya agotado lealmente todo su esfuerzo para que los particulares asuman tales actividades (Fermandois, 2000, p.73).

El mencionado principio tiene reconocimiento en el ordenamiento jurídico chileno en el más alto nivel, particularmente en el artículo 1 de la Constitución, el cual señala que el Estado reconoce y ampara a los grupos intermedios, garantizando su adecuada autonomía para cumplir sus fines específicos, estableciendo en el inciso siguiente la concepción de un Estado al servicio de la persona humana, el cual debe crear las condiciones para que cada integrante de la comunidad logre su mayor realización espiritual y material posible. Por su parte, el artículo 19, numeral 21, fija el derecho de las personas a desarrollar cualquier actividad económica y limita la actividad empresarial del Estado a solo cuando una ley de quorum calificado lo autorice, y en igualdad de condiciones con los particulares. Este último derecho goza de poderosas garantías, como son la acción constitucional de protección y la acción de amparo económico.

A partir de esta forma de entender la función del Estado, surge la lógica consecuencia de que la autoridad no debe intervenir en las actividades que desarrollan los particulares, y en ciertos casos, hacer un esfuerzo tendiente a permitir la actuación de estos, creando condiciones que faciliten cumplir sus fines particulares y la satisfacción del interés general o bien común. Así las cosas, en ciertos mercados, como el energético, en el cual -producto de sus peculiaridades- no se puede permitir una absoluta libertad sin afectar el bien común, el Estado, antes de intervenir dando la prestación directamente, debe crear condiciones que permitan a los particulares lograr su objetivo de manera eficiente, he ahí la labor que le corresponde a la regulación económica.

En este sentido, tal como señala Vergara Blanco, el marco fijado por la Constitución chilena prescribe que las actividades de servicio público con carácter industrial o económico pueden y deben ser desarrolladas ab initio por los particulares en aplicación del principio de subsidiaridad (2004, p. 68). A partir de este aspecto fundamental de nuestro orden público económico, es posible entender cómo se materializó este principio en la provisión del servicio eléctrico en el país, mediante fundamentales y radicales reformas que son conocidas como de liberalización del mercado eléctrico, que dan paso de un suministro prestado por el Estado a uno dado por particulares, en mayor o menor medida, bajo condiciones de competencia.

En un sentido amplio, la liberalización supone el levantamiento de barreras y condiciones establecidas por el poder público para la participación de la iniciativa privada en un sector económico (Caballero, 2011, pp. 45-46) o, como dice Ariño, es abrir mercados y romper monopolios (2010, p. 138). Lo anterior no significa que se levantan todas las barreras, sino que el Estado deja de prestar un determinado servicio 
de forma directa para dar paso a los particulares en condiciones propias de mercado, pero sujeto a ciertas obligaciones y limitaciones en virtud del interés público relevante existente en la actividad.

En el caso de los servicios públicos, como la electricidad, cambia el papel asignado al Estado, pues si bien sigue interesado en asegurar la prestación de los mismos en beneficio de los consumidores, ya no lo presta de manera directa, sino indirecta, por una parte facilitando la existencia de competencia, mientras que por otra, en aquellos casos en que la competencia no existe por cualquier motivo, evitando las perniciosas consecuencias de su falta mediante la imposición de obligaciones jurídicas efectivas (Montero, 2013, pp. 83-84).

En definitiva, el Estado pasa a ser un garante de la prestación del servicio, vigilando el comportamiento de los mercados para que los operadores respeten la competencia y cumplan sus obligaciones de servicio público (Muñoz, 2009, pp. 17-18).

El fundamento económico, que justifica mantener una regulación de la actividad y no entregar una plena libertad, se encuentra en que, en ciertas actividades, como la transmisión y distribución eléctrica, se presentan fallas en el mercado que ameritan una regulación estatal que impida abusos o afectaciones al bien común.

De este modo, el ideal en las economías modernas en un régimen de libertad y competencia es que el intercambio económico genere transformaciones que mejoren el bienestar de una persona sin empeorar el de otra, lo que en ciencia económica se conoce como óptimo de Pareto (Stiglitz, 2000, p. 69). Sin embargo, el beneficioso equilibrio antes descrito no siempre se logra, de manera tal que, cada vez que en una actividad no se alcance el óptimo paretiano, se producen los denominados fallos de mercado, que van a justificar la intervención del Estado (De la Torre, 2014, p. 49).

Estos fallos pueden ser de distinto tipo, a saber, de competencia, de mercados incompletos, de información, por externalidades, entre otros. Dentro de estos, para efectos de este trabajo, es particularmente relevante el fallo de competencia y poder de mercado ${ }^{4}$. Lo anterior por cuanto el mercado eléctrico, en muchos aspectos, se presenta como un monopolio natural, y se define como aquel en que los costos de producción son tales que para los consumidores les sea más barato obtener el producto de una empresa que de varias, lo anterior en razón de que no resulta conveniente

4 Existe poder de mercado cuando alguna de las empresas, de forma individual, o algunas de las empresas, de forma colectiva, disponen de la habilidad de tomar acciones estratégicas que alteren el equilibrio del mercado con el objeto de aumentar sus beneficios por encima de su nivel competitivo Fabra (2004, p. 202). 
una duplicación ineficiente, por tratarse de economías de escala (De la Torre, 2014, p. 50) $)^{5}$. Esto se aprecia en las redes de transmisión y distribución, donde no resulta conveniente insertar competencia. La existencia de fallos de competencia aleja de los óptimos paretianos, que se traduce en que los particulares por sí solos, en un régimen de libertad, no pueden cumplir de manera satisfactoria con la prestación de un servicio, lo que es crítico en aquellos servicios de interés general, como es el suministro de electricidad.

En virtud de lo antes expuesto, se hace palpable la justificación de la intervención estatal en este mercado liberalizado a través de sus diversas regulaciones orientadas a permitir la competencia y aminorar las fallas de mercado.

\section{INTEGRACIÓN VERTICAL Y SEPARACIÓN DE ACTIVIDADES}

La integración vertical ha sido un tema de amplia discusión en los procesos de privatización y liberalización del sector eléctrico, particularmente si se pretende crear condiciones de competencia en segmentos que son potencialmente competitivos.

En general, la teoría económica entiende la integración vertical como una falla de mercado, la cual es vista de dos formas, tanto como una respuesta a los problemas de un poder de mercado preexistente (por ejemplo, crear un monopolio estatal) o bien, como un movimiento estratégico de una empresa para crear o aumentar su poder de mercado, particularmente en mercados ascendentes y descendentes, como es el eléctrico (Joskow, 2005, p. 320).

Este fenómeno implica que una empresa integre en la acción productiva nuevas actividades complementarias relacionadas con el bien o servicio que presta, tanto por encima o por debajo de la cadena de producción, lo que puede ocurrir con la finalidad de reducir costos de producción y transacción, controlar suministros o lograr mayor calidad del bien (Tamayo y Piñeros, 2007, p. 33). Sin embargo, en el caso del sector eléctrico, un poderoso estímulo es tener el control del mercado.

Se debe tener en cuenta que en un mercado perfectamente competitivo donde las distintas fases del proceso productivo están sujetas a un régimen de competencia, la integración vertical no generará mayores problemas. Sin embargo, si en alguna fase del proceso existe una actividad monopólica, sin una adecuada separación de actividades, se dificulta enormemente dar cabida al mercado en los segmentos potencialmente

5 El autor explica la condición de monopolio natural en las economías de escala, en las cuales una única empresa puede producir un servicio o producto a un menor costo posible, esto es, a mayor producción menor costo. De este modo, si existen más empresas, necesariamente cada una tendrá un menor nivel de producción aumentando el coste medio Mankiw (2002, p. 199). 
competitivos. De cierta forma, quien posee la actividad monopólica, tiene la llave maestra de acceso al mercado.

Situación como la antes descrita se presenta en el mercado eléctrico, el cual, a lo menos en la regulación chilena, se divide en tres segmentos: generación, transporte y distribución 6 . La empresa integrada verticalmente es aquella que integra bajo su gestión las tres - o a lo menos dos- fases del suministro de electricidad, emulando, de cierta forma, las características de un sistema eléctrico completo (Fabra, 2004, p. 81).

Dentro de este esquema, los segmentos de transporte y distribución operan como red única y mantienen un carácter de monopolio natural, no así la generación, donde se privilegia la libertad de precios y la libre iniciativa para el emprendimiento, por así permitirlo la propia naturaleza de la actividad (Evans y Yáñez, 2017, pp. 1-2).

De esta forma, por muy competitivo que pueda ser el mercado de la generación, si alguno de los operadores controla la única y monopólica red de transporte les será imposible a los demás llegar a los usuarios finales, o si pueden llegar a hacerlo lo harán en condiciones desfavorables respecto del generador-controlador. Por tanto, en el sector eléctrico es fundamental la separación de las actividades potencialmente competitivas - la generación y, en algunos ordenamientos jurídicos la comercializa ción- de aquellas que no lo son, para así impedir que existan subsidios cruzados o un desmedido aumento del poder de mercado (De la Cruz, 2009, p. 295).

Sobre los subsidios cruzados, estos se pueden dar en empresas verticalmente integradas, donde las pérdidas de un servicio pueden ser financiadas por otro u otros servicios rentables (Ruiz, 2014; Muñoz, 2009, p. 35). En el caso del mercado eléctrico, esto podría ocurrir mediante un generador que vende su energía a un precio inferior al costo marginal, cuya diferencia se cubre con la mayor tarifa de la actividad regulada, lo que puede ser un fuerte incentivo para la competencia desleal (Alé, 1990, p. 177).

De este modo, el generador que tiene el control o incidencia relevante en el segmento de transporte de electricidad va a gozar de una posición dominante, esto es, una situación respecto de sus competidores que implica tener la capacidad de excluirlos de un mercado o contenerlos (Ybar, 2016, pp. 16-17). En particular, el factor principal para gozar de este dominio se lo entrega poseer o controlar la red única de transporte. Esta situación, en la que existe un agente económico que tiene una posición de dominio

6 En la legislación chilena no se reconoce la comercialización como un segmento distinto a la generación o distribución. Cosa distinta sucede en la liberalización del sector eléctrico europeo, en donde se reconoce la comercialización como un cuarto segmento, en virtud del cual el consumidor tiene el derecho a adquirir la energía en el mercado libre, a cualquier sujeto autorizado, pagando peajes de uso y acceso a las redes (Salinas, 2009, pp. 766-767). 
es particularmente peligrosa para los consumidores y la eficiencia del sector cuando se trata de un mercado relevante como es el eléctrico, donde no existe sustituibilidad de la demanda y el mercado es inelástico. Así las cosas, de producirse un abuso de posición de dominio en un mercado relevante, necesariamente nos encontramos con una conducta que infringe el régimen competitivo (Bustos, 2015, p. 116).

Así las cosas, resulta fundamental que la regulación económica separe las actividades competitivas de las que no lo son, pues esto permite reconocer a las primeras libertades de acceso al mercado, de intervención, de acceso a infraestructura y de contratación y formación competitiva de precios. Por otro lado, se requiere que actividades no competitivas queden sujetas a un estatuto jurídico que imponga su real gestión separada, que permita el uso abierto de los operadores, la abolición de derechos de exclusividad, la planificación por el regulador de las extensiones de red, la regulación de las tarifas de acceso y uso, como también imponer obligaciones de servicio universal y servicio público (De la Cruz, 2009, p. 295).

En definitiva, en atención a la finalidad de la regulación económica, lo buscado mediante la separación de actividades es evitar discriminaciones en el acceso a las redes por parte de empresas monopólicas -integradas verticalmente o no- que ejerzan un poder de mercado para favorecer a ciertas generadoras relacionadas 0 vinculadas (Sánchez, 2007, p. 79). Para lograr estos objetivos, el derecho ha venido interviniendo en esta actividad mediante distintos tipos o niveles de separación entre las actividades del transportista en empresas verticalmente integradas en el sector eléctrico. De esta forma, la normativa puede buscar la separación: i) jurídica, ii) contable, iii) funcional y iv) accionarial (Muñoz, 2009, p. 36).

Respecto a la separación jurídica, implica que cada actividad dentro del sector energético debe ser desarrollada por personas jurídicas distintas, pero no impide que un mismo grupo empresarial sea el propietario de cada una de estas entidades. En cuanto a la separación contable, refiere a que las distintas actividades que integran el sector deben llevar una contabilidad separada de las demás actividades que desarrollen, para así poder garantizar la transparencia. Por su parte, la separación funcional (iii) supone la adscripción de medios materiales y personales específicos y dotados de un cierto grado de independencia, de manera que los gestores de la red puedan hacer efectiva su independencia, sin participar en las demás actividades del grupo. Y, finalmente, la separación accionarial o patrimonial (iv) es el medio más radical de separación de actividades, por cuanto impide que los mismos propietarios o accionistas de empresas de algún segmento del sector energético constituyan o adquieran la propiedad o control de la empresa de transporte (Muñoz, 2009, p. 36; Guillén, 2010, pp. 298-299). 
Todas estas medidas, en definitiva, aspiran a otorgar independencia a los gestores de las redes de transporte (transmisión y distribución), condición fundamental para el desarrollo de la competencia en el sector eléctrico, pues solo así se puede garantizar un acceso a las redes en condiciones objetivas y no discriminatorias (Ariño, 2006, p. 72).

En atención a los fines buscados con estas medidas, la separación jurídica y la contable apuntan principalmente a impedir los subsidios cruzados entre el operador del sistema de transporte con las demás actividades del grupo de empresas al que pertenece el transportista (Muñoz, 2009, pp. 35-36). Lo anterior permite a la autoridad supervisar que se cumplan las normas relativas al derecho de la competencia (Guillén, 2010, pp. 298-299), evitando así prácticas abusivas, como vender la energía bajo el costo marginal y compensar dicha pérdida con las utilidades de la operación de la red de transporte.

Por su parte, la separación accionarial, si bien es, sin duda, el más efectivo mecanismo de separación de actividades, presenta grandes dificultades para aplicarse a empresas que ya se encuentran verticalmente integradas, pues implica una afectación directa a un derecho de propiedad adquirido ${ }^{7}$, siendo en consecuencia una medida sumamente onerosa para el Estado y muy resistida por los operadores. Lo anterior no obsta para limitar a los propietarios o accionistas de sectores sujetos a competencia poder adquirir la propiedad o acciones en empresas de transporte hacia futuro.

De esta manera, teniendo en consideración lo insuficiente que puede resultar una separación jurídica y contable, y por otro lado lo complejo y costoso que puede significar separar patrimonialmente un holding ya verticalmente integrado, el regulador se ve en la necesidad de buscar algún punto intermedio que se acerque lo más posible al fin buscado dentro de sus posibilidades. Es así como surge la técnica de la separación funcional a medio camino de una separación total, que permite asegurar de mejor forma la necesaria independencia de los operadores de los monopolios naturales del sector eléctrico. Esta separación busca, a través de una serie de prevenciones, garantizar la independencia organizativa y gerencial de la sociedad operadora de la actividad monopólica con giro exclusivo (Nebreda, 2008, p. 11).

Estas medidas y prevenciones son el complemento necesario para aprovechar los beneficios que reporta la separación jurídica de las actividades monopólicas en el sector eléctrico. De no ser así, si esta empresa separada y con giro único pertenece a un mismo grupo de empresas que operan sectores competitivos del mercado de la electricidad, los gerentes y administradores responderán ante los controladores del

7 En el caso español se nacionalizaron las redes de alta tensión, las cuales pasaron a ser propiedad de una sociedad estatal a la que se le atribuye la gestión del servicio. Sin perjuicio de ello, permitió a las empresas originalmente propietarias tener participación (Pajuelo, 2009, pp. 552-553). 
holding, quienes tendrán incentivos para exigir de estas prácticas tendientes a subsidiar otra actividad de la cadena, o entorpecer el ingreso de nuevos competidores.

Tales medidas de separación funcional no aseguran una absoluta autonomía e independencia de la empresa integrada verticalmente, pero, por lo menos, crean reglas claras y herramientas nuevas y efectivas para el ente fiscalizador, tendientes a perseguir y sancionar conductas que puedan afectar la independencia y autonomía de la empresa que opera una actividad monopólica.

Teniendo en cuenta las dificultades de la separación accionarial, y lo efectivas pero insuficientes que son la separación jurídica y contable, surge como una condición fundamental que exista una separación funcional entre los transportistas y demás actividades eléctricas de la empresa. Esto exige que se asegure, bajo un sistema de acceso regulado y publicitado, la independencia real de los gestores de redes (Ariño, 2006, pp. 72-73).

\section{LA SEPARACIÓN DE ACTIVIDADES EN LA UNIÓN EUROPEA}

Para conocer cómo se ha desarrollado la regulación económica de la separación de actividades en el sector eléctrico, el caso europeo, y en particular el español, sirve como gran ejemplo, ya que desde que se iniciaron los esfuerzos liberalizadores el romper estos grupos integrados ha ocupado un papel central, siguiendo un tránsito progresivo en la materia. Este paulatino y constante proceso se asemeja bastante al caso chileno, pues ambas regulaciones avanzan en una misma dirección, con la salvedad que esta última normativa aún va un paso más atrás.

Desde sus inicios, la Unión Europea ha buscado levantar las fronteras de las economías del continente y hacerlas converger en una verdadera unión económica - un mercado común europeo- que permita el libre intercambio en condiciones de competencia entre los actores de los distintos Estados miembros, para lo cual es fundamental alcanzar acuerdos que permitan homogenizar sus instituciones jurídicas y económicas. En este sentido, la primera Directiva europea tendiente a concretar este objetivo en el mercado eléctrico fue la 96/92/CE del año $1996^{8}$. Este instrumento buscaba sentar las bases de un avance progresivo y racional hacia un mercado interior de la electricidad, en el cual se permitiría la existencia de un mercado competitivo sin dejar de respetar las obligaciones de derecho público propias de la actividad.

Este instrumento define la organización y funcionamiento del sector eléctrico, para estructurar un mercado competitivo y no discriminatorio, garantizando el acceso de los distintos actores al mercado y reconociendo a su vez obligaciones de servicio público y protección medioambiental. 
La Directiva tiene el mérito de reconocer los problemas principales que presenta el proceso progresivo de liberalización del sector: esencialmente las características monopólicas de las actividades de transmisión y distribución, como también la fuerte integración vertical de las empresas eléctricas que realizan estas actividades (Olivares, 2014, p. 212). En este sentido, en su parte considerativa, la Directiva establece importantes lineamientos referidos a la separación de actividades, haciendo hincapié principalmente en la transparencia de las operaciones de empresas verticalmente integradas, como también la exigencia de llevar una contabilidad separada, para evitar prácticas discriminatorias que puedan importar un abuso de su posición dominante (véanse los considerandos 30, 31, 32 y 33 de la mencionada Directiva).

Ya en su articulado, en el capítulo VI, la Directiva da derecho a los Estados miembros y a los órganos competentes para acceder a la contabilidad de las empresas del sector eléctrico para efectos de ejercer su labor de control. Además, también se obliga a las empresas integradas verticalmente a llevar contabilidad en cuentas separadas, para evitar discriminaciones, subvenciones cruzadas y falseamiento de competencia (ver los artículos 13, 14 y 15).

Como se puede apreciar, la Directiva europea 96/92/CE no obligaba a la separación jurídica de las redes de transporte, lo que dio lugar a denuncias por el abuso de las compañías verticalmente integradas (De la Cruz, 2009, p. 295). En definitiva, solo se consagró una separación contable, descartándose así las otras formas de separación, como son la funcional, accionarial y jurídica. Sin perjuicio del importante paso que significó para la liberalización del sector esta normativa, el Parlamento Europeo consideró modernizar su regulación con miras a mejorar el funcionamiento y superar las deficiencias que aún presentaba el mercado interior de la electricidad. Así, se dictó la Directiva 2003/54/CE con la finalidad de garantizar condiciones equitativas en el ámbito de la generación, reducir el riesgo de que aparezcan posiciones dominantes o de comportamiento abusivo y garantizar el acceso a las redes de transporte mediante una gestión independiente y no discriminadora (considerandos 1, 2 y 3).

Sobre la separación de actividades, la normativa del 2003 mantuvo la necesidad de la separación contable de las empresas de transporte respecto a las demás actividades del grupo, pero, además, con miras a garantizar la independencia del gestor de las redes de transporte, estableció la obligación de contar con una personalidad jurídica propia y una organización y toma de decisiones independiente de la empresa verticalmente integrada. Para lograr esta independencia, las personas responsables de la administración de la red no podían participar en estructuras de la empresa verticalmente integrada, además de contar con poder de decisión efectivo e independiente. Por otro lado, se debían establecer mecanismos para evitar que información confidencial del gestor de red pasara a otras partes de la empresa, pero conservándose mecanismos 
de coordinación, de control financieros y de gestión (cf. Artículo 10 de la Directiva 2003754/CE y ver Sánchez, 2007, pp. 74-78). De este modo, esta Directiva mantiene la separación contable, incorpora una separación jurídica, y de una manera muy tímida incorpora una separación funcional al otorgar cierto poder de decisión al operador de red, pero sujeto a un control financiero y de gestión respecto al grupo de empresa.

Actualmente, el mercado interior de la electricidad en la Unión Europea está regulado por la Directiva 2009/72/CE, que busca continuar con el desarrollo de los principios básicos del proceso liberalizador, el acceso de los terceros a la red y la separación de las empresas verticalmente integradas (Olivares, 2014, p. 212). Este instrumento, en su parte considerativa, reconoce que mientras no exista una separación efectiva entre las redes y las actividades de generación y suministro existe un riesgo intrínseco de discriminación. Acto seguido, indica que las técnicas de separación jurídica y funcional establecidas en la directiva anterior (2003/54/CE) no han logrado una separación efectiva entre estas actividades (considerandos 9 y 10).

Por su parte, para avanzar en una efectiva separación de actividades se indican dos métodos. El primero -y considerado más eficaz- es una separación patrimonial o accionarial de la actividad de transporte, en virtud de la cual una misma persona o grupo de personas no puede ejercer control sobre una empresa de generación o de suministro, y al mismo tiempo ejercer control o cualquier derecho sobre un gestor de la red de transporte y viceversa (considerandos 11, 14, 15 y artículo 1 de la Directiva 2009/72/CE, 4). Un segundo mecanismo que contempla la Directiva (considerandos 16 , 19,23 , y artículos 9, 10, 11, 12 y 13) -para el caso en que no se haga una separación patrimonial- es la creación de un gestor de red realmente independiente, libre de los intereses del grupo empresarial, para lo cual se debe establecer una regulación detallada y mecanismos de control amplio que permitan cumplir con esta exigencia.

En definitiva, esta última directiva europea recomienda una separación mayor de actividades mediante una separación patrimonial o accionarial, para impedir cualquier tipo de control sobre los gestores de redes. Sin embargo, considerando las dificultades que podría tener este proceso, establece la posibilidad de avanzar hacia una real separación funcional de las actividades de transporte, para lo cual la regulación debe consagrar mecanismos y reglas que permitan una real independencia de las personas que administran las redes.

Las Directivas mencionadas son vinculantes para los Estados miembros de la Unión Europea, quienes deben adaptar su normativa interna con miras a satisfacer las exigencias comunitarias y crear las condiciones para el desarrollo de un mercado interior de la electricidad. En este sentido, el caso español es un buen ejemplo de cómo se han incorporado progresivamente las recomendaciones hechas por las dis- 
tintas directivas a su legislación interna, particularmente en materia de separación de actividades. Así, el proceso liberalizador del mercado de la electricidad en España fue iniciado por la Ley 54/1997, la cual fue dictada con el propósito de incorporar en el orden interno las previsiones contenidas en la Directiva 96/92/CE.

Esta ley, en lo que respecta a la separación de actividades (artículos 14 y 20), establece que las sociedades que se dediquen a actividades reguladas (transporte) deben tener como objeto exclusivo el desarrollo de las mismas, no pudiendo participar en las actividades de generación o comercialización. Sin embargo, permite que un grupo de empresas puedan participar en actividades reguladas y no reguladas, pero solo en una de ellas de manera directa, y en las demás solo mediante la titularidad de acciones. Por su parte, también se exige a las distintas empresas del grupo mantener contabilidad separada. De esta forma, la normativa española de ese entonces cumplía holgadamente con las exigencias que hacía el derecho comunitario, pues fijaba una separación jurídica y contable de las actividades reguladas y no reguladas de un mismo grupo empresarial. Sin embargo, no establecía mecanismo ni técnicas para permitir una separación funcional.

Esta situación se mantuvo hasta el año 2007, cuando mediante la Ley 17/2007 se modificó, sin derogar, la Ley 54/1997, para incorporar las nuevas directrices comunitarias establecidas en la Directiva 2003/54/CE. Esta medida profundizó el sistema de separación de actividades en el sector eléctrico español, avanzando hacia una separación funcional y de gestión entre las diferentes actividades. La modificación estableció una serie de exigencias a los grupos de sociedades que desarrollen actividades incompatibles, en las que además de la separación jurídica y contable, deben cumplir ciertos criterios de independencia en la gestión de las redes. Dentro de las principales exigencias se encuentra la prohibición de que las personas responsables de esta gestión puedan participar en las estructuras organizativas del grupo de empresas o de las actividades de generación o comercialización; protección en cuanto a retribución y cese de personas que participen en segmento transporte; prohibición de compartir información sensible entre las distintas empresas del grupo; capacidad de decisión efectiva de los administradores de las redes, entre otras. Además, para el cumplimiento de estos requisitos, la sociedad que realiza la actividad regulada deberá tener un código de conducta que exponga medidas para garantizar el cumplimiento de las mismas (ver la Ley n. ${ }^{\circ}$ 17/2007, artículo único). Sin perjuicio de lo anterior, si bien la legislación española permitió que grupos de empresas pudieran ejercer actividades reguladas y no reguladas cumpliendo con las exigencias de separación contable, jurídica y funcional, estableció un sistema progresivo para llegar a una separación accionarial o patrimonial. De este modo introdujo la figura del transportista único, para lo cual las distintas empresas propietarias de redes de transporte deberán vender a la Red Eléctrica de España S. A. (empresa estatal) sus activos, así, pasa a ser esta entidad 
la única propietaria y gestora de la red (Pajuelo, 2009, pp. 558-560; Ley n. ${ }^{\circ}$ 17/2007, disposición transitoria novena).

Este sistema de separación de actividades luego es mantenido por la nueva ley del sector eléctrico, de manera que Red Eléctrica de España, S. A., actúa como transportista único desarrollando la actividad en régimen de exclusividad (artículos 12, 20 y 34).

\section{SISTEMA CHILENO DE SEPARACIÓN DE ACTIVIDADES: PROBLEMAS Y PROPUESTAS}

\subsection{Modelo de separación de actividades introducido por la Ley Corta I}

La mayor y más integral reforma al sector eléctrico en el mundo, posterior a la Segunda Guerra Mundial fue la operada en Chile. Esta fue concebida desde 1978 con la creación de la Comisión Nacional de Energía, materializándose finalmente en 1982 con la expedición de la Ley General de Servicios Eléctricos (LGSE). Esta normativa fue un cambio de paradigma que privatizó y liberalizó el mercado casi una década antes de las reformas al sector eléctrico en Inglaterra y Gales?.

Esta pionera ley -Decreto con Fuerza de Ley N. 1 de 1982 del Ministerio de Minería-, si bien reguló las actividades de los distintos sectores en diferenciados segmentos de generación, transporte y distribución, nada dijo sobre la integración vertical del sector. Así las cosas, se instauró una práctica en la que los peajes por uso de las redes que pagaba cada generador eran determinados de manera bilateral y diversa, lo que naturalmente provocó discriminaciones al momento de distribuir los costos de transmisión entre los distintos usuarios (Evans y Yáñez, 2017, p. 271). Esta situación legislativa que toleraba la integración vertical -una cortapisa para un mayor desarrollo del mercado eléctrico - fue denunciada tanto por la Fiscalía Nacional Económica como por las empresas no propietarias de las redes de transporte, las cuales solicitaron a la Comisión Resolutiva Antimonopolios (antecesora del Tribunal de la Libre Competencia) que dispusiera "desconcentrar y descentralizar las actividades de generación, transmisión y distribución (...) mediante la creación de empresas con patrimonios independientes y administración separada" tal como consta en la Resolución n. ${ }^{\circ} 488$ de 11 de junio de 1997, visto 4, esto es, que se procediera a separar jurídica y funcionalmente estas actividades (Serra, 2011, pp. 447-448).

Sin embargo, la Comisión desechó los requerimientos por considerar que la estructura de propiedad que presentaban en ese entonces las empresas dominantes no era, por sí sola, un factor relevante y determinante que afectase la competencia o que tendiera a ello, siempre que se asegurara en la legislación la libre entrada a la actividad y condiciones homogéneas para todos los participantes en los segmentos no

Sobre el origen e impacto de la reforma al sector eléctrico en Chile, ver Pollitt (2004, pp. 2-8). 
competitivos del mercado -Resolución n. 488 de 11 de junio de 1997, considerando vigesimoséptimo-. Situaciones como la antes descrita generaron un profundo replanteamiento del modelo regulatorio que databa de 1982. Esto llevó a una importante reforma legal, la Ley 19.940, conocida como Ley Corta I.

Tal como señala el mensaje que envió el ejecutivo, esta reforma reconoce que el sistema vigente de cobro de peajes - sumado a que la ley no imponía limitación alguna a la integración vertical transmisión-distribución-generación-permitía que se pudiera hacer un uso discriminatorio de las redes como herramienta de competencia desleal, lo que evidentemente era un riesgo que desincentivaba las inversiones (Serra, 2011, p. 449; Biblioteca del Congreso Nacional, 2004).

La reforma, entre otras cosas, tuvo la virtud de introducir la noción de separación de actividades en sector eléctrico chileno, a través de los nuevos incisos que incorporó al artículo 7 de la LGSE. En concreto, estableció la obligación para las empresas operadoras o propietarias del sistema de transmisión troncal de constituirse como sociedades anónimas abiertas, prohibiendo a estas personas jurídicas dedicarse al giro de generación y distribución (artículo 4). Por otra parte, en cuanto a la propiedad de estas sociedades dedicadas exclusivamente al transporte de electricidad, la Ley 19.940 en el artículo 4, estableció un límite a la participación que podrían tener otras sociedades o personas dedicadas a otro segmento del sistema eléctrico. De esta manera, el límite a la participación individual no podría exceder del 8 \% de la inversión total del sistema de transmisión troncal ${ }^{10}$, mientras que la participación conjunta de estos sujetos no podía exceder del 40 \% de la inversión total. Cabe agregar que esta limitación solo opera a futuro.

En síntesis, esta reforma estableció nítidamente una separación jurídica y contable entre las actividades de transmisión y las demás del sistema eléctrico, lo que evidentemente significa un avance, sobre todo para evitar subsidios cruzados y permitir a los entes fiscalizadores el acceso a información que pueda evidenciar una práctica anticompetitiva o desleal.

Tratándose de separación patrimonial o accionarial, la normativa chilena es muy incipiente, por cuanto solo la regula hacia inversiones posteriores al año 2004, además, las limitaciones establecidas para impedir que una empresa verticalmente integrada adquiera el control de una sociedad de transmisión, son respecto a la totalidad del sistema de transmisión nacional, así podría adquirir el control en ciertos segmentos de las redes.

10 El sistema de transmisión troncal pasó a llamarse "sistema de transmisión nacional" debido a las reformas introducidas por la Ley n.o 20.936 del año 2016. 


\subsection{Reforma de la Ley n.' 20.936: acceso abierto y nueva institucionalidad}

Un aspecto central para el buen funcionamiento de un mercado donde existen segmentos con naturaleza de monopolio natural es que concurra la posibilidad de que todos los actores de sectores competitivos tengan acceso a este segmento de manera libre y en condiciones no discriminatorias. Para tal efecto, en el mercado eléctrico se establece el principio de acceso abierto a las redes, lo cual implica permitir a los distintos generadores tener acceso a la infraestructura en condiciones técnicas y económicas no discriminatorias, pagando remuneración regulada, y se prohíbe a sus operadores establecer restricciones injustificadas o favorecer a ciertos generadores, lo que distorsionaría la competencia del mercado (Schnake, 2015, pp. 59-61; LGSE, artículo 79).

Si bien el acceso abierto fue incorporado a la regulación eléctrica chilena mediante la Ley Corta I, esta presentaba falencias que impedían el cumplimiento de su finalidad. Entre los problemas que se señalaban para ejercer el acceso abierto se destaca que se debía negociar directamente con el propietario de las instalaciones, restando toda imparcialidad; no se establecía dónde debían ser los seccionamientos de la red; no se garantizaba el derecho en la transmisión adicional; además, se generaban muchas controversias e interpretaciones que afectaban la seguridad de los inversionistas (Biblioteca del Congreso Nacional, 2016) ${ }^{11}$.

En este sentido, la Ley n. ${ }^{\circ} 20.936$ hizo importantes modificaciones a la regulación del segmento transmisión tendientes a mejorar el acceso abierto. Tratándose del sistema de transmisión nacional, la normativa establece la obligación al operador de la red de permitir la conexión a sus instalaciones sin discriminaciones, no pudiendo negarla por motivos de capacidad técnica. Para estos efectos, se establece un proceso de conexión donde se toman en cuenta las observaciones planteadas por las partes sobre las que debe hacerse cargo el coordinador en la autorización de conexión. Por su parte, se establece la obligación de los operadores de las instalaciones de dar todas las facilidades necesarias para que terceros ejecuten las obras (LGSE, artículo 79).

En lo que respecta a los sistemas de transmisión dedicados ${ }^{12}$, el acceso abierto no goza de las mismas garantías que la conexión al sistema de transmisión nacional. La principal deficiencia se presenta porque, si bien los operadores siguen estando obligados a no negar el servicio, esto estará sujeto a que la red tenga capacidad técnica disponible, la cual será determinada por el coordinador. Por su parte, en caso de existir

11 Sobre las deficiencias del sistema de acceso abierto establecido desde la Ley Corta I (Schnake, 2015, pp. 59-73).

12 Se trata de redes eléctricas que se encuentran interconectadas al sistema eléctrico, dispuestas para proveer de energía a clientes no sometidos a regulación de precios o para inyectar producción de generadoras al sistema eléctrico. Artículo 76 de la LGSE. 
discrepancias en relación con la aplicación del régimen de acceso abierto, se puede solicitar la intervención del panel de expertos para resolver los aspectos técnicos y económicos. Mientras que, tratándose de fiscalizar el cumplimiento de la normativa, lo resuelto por el Coordinador o por el mismo panel de expertos, corresponde la actuación de la Superintendencia de Electricidad y Combustibles (SEC).

Otro aspecto relevante de la Ley n. ${ }^{\circ} 20.936$, en los artículos 87 y siguientes, es la regulación del plan de expansión del sistema de transmisión. Este consiste en una planificación anual de la Comisión Nacional de Energía, que debe considerar las obras de expansión, ampliación y nuevas obras necesarias para el sistema de transmisión nacional, de polos de desarrollo, zonal y dedicados. En este proceso pueden participar los distintos interesados del sector, finalmente se obtiene como producto un decreto de expansión de la transmisión, o para obras nuevas, según el caso. Con ello, se debe iniciar una licitación pública internacional para realizarlas.

Por su parte, para la correcta coordinación del sistema, el monitoreo de la competencia y para garantizar el ejercicio del derecho al acceso abierto, la ley (artículos 212-1 y siguientes) creó una nueva figura, el Coordinador Independiente del Sistema Eléctrico Nacional, una corporación autónoma de derecho público, como un organismo técnico e independiente encargado de velar por estas materias (Evans y Yáñez, 2017, pp. 306-308).

A esta institución, aparte de su función como coordinadora del mercado eléctrico y transferencias económicas entre las empresas sujetas a su coordinación, le corresponden importantes roles respecto al acceso abierto y al monitoreo de la competencia. El acceso abierto debe garantizarlo mediante la autorización a la conexión a los sistemas de transmisión por parte de terceros, verificando el cumplimiento de los requisitos y exigencias e instruyendo las medidas necesarias, además de determinar la capacidad técnica disponible en los sistemas de transmisión dedicados y autorizar el uso de dicha capacidad.

En lo que respecta al monitoreo de la competencia, la Ley (artículo 72-10) impone a esta institución la obligación de monitorear permanentemente las condiciones de competencia existentes en el mercado eléctrico, y para el caso de detectar indicios de actuaciones que podrían de alguna forma llegar a ser atentados contra la libre competencia, debe ponerlas en conocimiento de la Fiscalía Nacional Económica o la autoridad que corresponda. Como se puede apreciar, las distintas innovaciones que vino a hacer la Ley n. 20.936 tienen una clara finalidad de atenuar los efectos adversos de la integración vertical, pero sin avanzar en una separación funcional ni mucho menos patrimonial. Así las cosas, llegados a este punto se justifica preguntar si logra sanear esta falla de mercado, o bien, si este importante avance puede ser 
profundizado mediante una mayor separación de los operadores de las redes de los demás actores sometidos a régimen de competencia.

\section{3 ¿Es necesario avanzar hacia una separación funcional?}

A pesar de lo reconocidos y estudiados que son los efectos perniciosos de la integración vertical en mercados monopólicos como el transporte de electricidad, no es posible sostener que es unánime la postura de que una mayor separación en el mercado eléctrico será beneficiosa para el sector y los consumidores, sobre todo en virtud de los avances que supuso la nueva ley de transmisión. En este sentido, Riesco y Vial (2015, pp. 43-56) ponen en entredicho la necesidad de mantener las prohibiciones de integración vertical del artículo 7 de la LGSE por no ser necesarias en el contexto actual. Así, postulan que esta norma ya no sería eficiente por varios motivos, entre los cuales destacan un cambio de la composición del mercado con el ingreso de nuevos actores al sistema transmisión, las garantías normativas e institucionales para materializar un acceso abierto y no discriminatorio, el plan de expansión de las redes en manos de la autoridad y por licitaciones públicas, y las facultades del coordinador y los entes de fiscalización.

Por su parte, en atención a que la integración vertical no es per se perjudicial, postulan que con un buen marco regulatorio e institucional no es necesario mantener la normativa que la prohíbe, lo cual permitiría aprovechar los potenciales beneficios que tendría la integración tanto para quienes integran el mercado como para los consumidores. En este sentido, destacan las eficiencias en la toma de decisiones como también la evitación de una doble marginalización, impedir aprovechamientos de los esfuerzos de otros agentes y la certidumbre que puede dar a las empresas, sobre todo por los altos costos de inversión (Riesco y Vial, 2015, pp. 46-48). Ante estos atendibles postulados, si bien sus premisas son correctas, desde ya se descarta que tengan la entidad y sean suficientes como para dar marcha atrás respecto a la separación de actividades en nuestro mercado eléctrico.

Evidentemente, existe un cambio sustancial en la composición del mercado eléctrico que ha permitido avanzar en una real separación de actividades, más aún, existen grupos de empresas integradas con participación en generación y transporte de electricidad (por ejemplo, el grupo Enel participa con un 21 \% de la generación eléctrica total del país, posee el $10 \%$ de las redes de distribución y un $2 \%$ en transporte) ${ }^{13}$. Además, se debe tener en cuenta que el motivo fundamental para que operara este cambio en el mercado fue la regulación de la separación de actividades que introdujo la Ley Corta I.

13 Sobre composición del mercado eléctrico chileno (Empresas Eléctricas A. G., 2018, pp. 9-14; Generadoras de Chile, 2018, p. 13). 
Cabe agregar que por mucho que existan hoy en día múltiples actores en el transporte de electricidad, esto en ningún caso significa que esta actividad dejó de ser monopólica, pues cada uno de los propietarios de los distintos segmentos de la red posee un monopolio en su respectivo tramo, no teniendo ningún otro límite que la regulación vigente para ejercer su poder de mercado. Es relevante tener en cuenta que el ingreso de nuevos actores al segmento transmisión -que redujo en alto grado de concentración de las empresas verticalmente integradas- es gracias, precisamente, a las exigencias que estableció la Ley Corta I, de manera que de no existir la prohibición del artículo 7 bien podría consolidarse una nueva concentración en el mercado. Por su parte, aunque la garantía del acceso abierto, los planes de expansión y los roles del coordinador y los entes fiscalizadores constriñen de manera importante las prácticas anticompetitivas que acarrea la integración vertical, no logran conjurarlas del todo.

Así, el Coordinador Independiente del Sistema Eléctrico Nacional, en presentación hecha en la causa Rol ERN-24-2018 del Tribunal de Defensa de la Libre Competencia, señala los potenciales riesgos que aún persisten en el sistema. Por un lado, la labor de los entes de fiscalización y coordinación puede ser dificultada producto de las asimetrías de información, ya que los fiscalizados, con intereses en otro segmento, tendrán un incentivo para no otorgar toda la información necesaria que pueda perjudicarlos y también podrían negar acceso a información clave, obligando a levantar procesos, inspecciones y auditorias, causando un aumento excesivo del tiempo de conexión. Otro riesgo lo representa el hecho de que el transmisor podría compartir información sensible de sus competidores con su parte relacionada en el segmento generación, dando lugar a un escenario propicio para conductas excluyentes.

En la misma causa indicada anteriormente, la Asociación Chilena de Energías Renovables y Almacenamiento señala las dificultades que presenta el acceso abierto en la legislación chilena, de las cuales destacan lo complicado que es tener acceso a la información, la complejidad del proceso de conexión y operación; todo ello se vería más agravado de no existir normativa que prohíba la integración vertical. Es por lo antes expuesto que se debe descartar cualquier modificación regulatoria que implique levantar las barreras que ya se han levantado contra la integración vertical.

Dicho esto, queda dilucidar si fuera de mantener las limitaciones ya existentes en esta materia, es necesario avanzar hacia una mayor separación de actividades para acorralar aún más las posibles prácticas anticompetitivas, en atención a que todavía subsisten empresas integradas verticalmente, y para el caso que puedan, en el futuro, relacionarse otras empresas del sector. Es por esta razón que se evidencia como una importante deficiencia de la regulación de separación de actividades chilena, la total inexistencia de mecanismos que permitan garantizar una real separación funcional 
en la administración de las empresas de transmisión y distribución que pertenezcan a grupos de empresas verticalmente integradas.

Nada impide que desde un grupo de empresas se puedan impartir órdenes e instrucciones a los administradores de las personas jurídicas dedicadas al segmento transmisión y distribución; no hay mecanismos de protección de la independencia de los administradores, no hay poder de decisión efectivo ni códigos de conducta que sancionen las intervenciones. De esta forma, mientras existan empresas en el sector de transporte que tengan intereses en la generación, van a existir incentivos para favorecerlas, de manera que los controladores esperarán de sus gerentes políticas que favorezcan al grupo, aun a costa de recurrir a conductas anticompetitivas.

Es por lo anterior -y teniendo en consideración las dificultades económicas y políticas de una separación accionarial- que un real e importante avance para el mercado eléctrico es incorporar mecanismos que garanticen una separación funcional entre las sociedades que controlan los sistemas de transmisión y distribución respecto de los grupos empresariales a los que pertenecen.

Para estos efectos, es altamente recomendable que el legislador incorpore a la LGSE técnicas y mecanismos como los establecidos en el artículo 12 de la Ley 24/2013 que regula el sector eléctrico español, tales como la prohibición de que las personas responsables de la gestión de las redes puedan participar en las estructuras organizativas del grupo de empresas o de las actividades de generación o distribución; protección en cuanto a retribución y cese de personas que gestionen la red; prohibición de compartir información sensible entre las distintas empresas del grupo; capacidad de decisión efectiva de los administradores de las redes; obligación de establecer un código de conducta que contemple medidas para garantizar el cumplimiento de las exigencias de separación funcional, entre otras medidas que se podrían implementar.

A pesar de que se ha sostenido que estas medidas serían ingenuidades, por cuanto estos holdings recurrirían a distintas artimañas para burlar las limitaciones impuestas por la regulación (Nebreda, 2008, p. 12), su implementación puede reportar significativos avances, debido a que se le entregará un contraincentivo importante a la empresa integrada para evitar conductas anticompetitivas -ya que acarrearía importantes sanciones- y daría a los entes fiscalizadores un escenario más propicio para ejercer su control, por cuanto por el solo hecho de infringir la normativa se configuraría el ilícito.

Para finalizar, cabe agregar que por muy loable que sean los objetivos planteados por la separación de actividades, cualquier medida requiere un profundo estudio, tanto de su alcance como de su impacto, por cuanto la experiencia ha demostrado que los 
efectos de la regulación sobre el mercado muchas veces pueden ser impredecibles (Sánchez, 2007, p. 85).

\section{CONCLUSIONES}

La integración vertical entre las empresas propietarias o gestoras de las redes de transporte con empresas dedicadas a otros segmentos del sistema eléctrico es una falla de mercado que presenta el sector eléctrico en sistemas liberalizados, la cual debe ser subsanada mediante técnicas de regulación económica.

El problema principal -que implica que los gestores de las redes pertenezcan a un grupo de empresas verticalmente integrado- radica en que este grupo tendrá un poder de mercado tal que le permitirá utilizar herramientas propias de competencia desleal, discriminando en el acceso a las redes, otorgándose subsidios cruzados con demás empresas del grupo y favoreciendo a compañías relacionadas.

Para solucionar este problema y lograr que exista un efectivo y no discriminatorio acceso a las redes, es fundamental la separación de actividades en las empresas verticalmente integradas en sus distintas modalidades, separando contable, jurídica y funcionalmente los grupos de empresas y, si es posible, también en lo patrimonial o accionarialmente.

En el caso chileno, la LGSE solo contempla una separación contable y jurídica, no existiendo mecanismos ni técnicas regulatorias que permitan una separación funcional que garantice una operación independiente de las redes respecto al grupo de empresas a que pertenece.

Es por esto que se hace necesario incorporar a la regulación económica del sector eléctrico obligaciones, prohibiciones y mecanismos para las empresas propietarias de redes de transmisión y distribución integradas a algún grupo de empresas que permitan garantizar la independencia y transparencia en su gestión, siguiendo como modelo el sistema español.

\section{REFERENCIAS}

Alé, J. (1990). Un nuevo esquema de regulación de monopolios naturales. Estudios Públicos, (37), 165-226.

Ariño, G. (2010). Problemas de competencia en los sectores energéticos. En M. Serrano y M. Bacigalupo (Coords.), Cuestiones actuales del derecho de la energía: regulación, competencia y control judicial (pp. 135162). Madrid: CNE-Iustel.

Ariño, G. (2006). Energía en España y desafío europeo: seguridad, concentración empresarial, competencia, regulación. Granada: Comares. 
Bascuñán, A. (1997). Tratado de derecho constitucional (Tomo IV). Santiago de Chile: Editorial Jurídica de Chile.

Betancor, A. (2010). Regulación: mito y derecho. Desmontando el mito para controlar la intervención de los reguladores económicos. Navarra: Civitas.

Biblioteca del Congreso Nacional. (2004). Historia de la Ley 19.940. Recuperado de https://www.bcn. cl/historiadelaley/nc/historia-de-la-ley/5720/

Biblioteca del Congreso Nacional. (2016). Historia de la Ley 20.936. Recuperado de https://www.bcn. cl/historiadelaley/fileadmin/file_ley/5129/HLD_5129_0948dlaf451123cf22b5db08a7adc19d.pdf

Bustos, M. (2015). El abuso de posición de dominio en el régimen jurídico español. Revista CES Derecho, $6(1), 111-122$.

Caballero, R. (2011). Las técnicas de regulación de la competencia, para la competencia y contra la competencia, y su contexto. En J. Santamaría (Dir.) y R. Caballero (Coord.), Las técnicas de regulación para la competencia: una visión horizontal de los sectores regulados (pp. 39-70). Madrid: Iustel.

Comisión Resolutiva Antimonopolios. Chile. (1997). Resolución 488/97 de junio 11. Recuperado de https://www.fne.gob.cl/biblioteca/jurisprudencia/decisiones-comisiones-antimonopolio-2/

Darnaculleta, M. (2009). La recepción y desarrollo de los conceptos y fórmulas de la regulación. El debate en la República Federal Alemana. En S. Muñoz y J. Esteve (Dirs.), Derecho de la regulación económica (Vol. 1) (pp. 349-386). Madrid: Iustel.

De la Cruz, J. (2009). El funcionamiento del sistema eléctrico. Sujetos. Separación de actividades. Planificación. En S. Muñoz y J. Esteve (Dirs.), Derecho de la regulación económica (Vol. 3) (pp. 279-332). Madrid: Iustel.

De la Torre, D. (2014). Fallos del mercado y regulación económica en los servicios públicos domiciliarios: aproximación a una disciplina poco estudiada por los juristas. Revista Digital de Derecho Administrativo, (12), 45-62.

Empresas Eléctricas A.G. (2018). Reporte eléctrico: transmisión y distribución. Recuperado de https:// www.electricas.cl/wp-content/uploads/2018/05/eelectricas-boletin-2018-05.pdf

Evans, E. y Yáñez, E. (2017). Derecho y regulación económica de la energía eléctrica. Santiago de Chile: Thomson Reuters.

Fabra, J. (2004). ¿Liberalización o regulación? Un mercado para la electricidad. Madrid: Marcial Pons.

Fermandois, A. (2000). Derecho constitucional económico (Tomo I). Santiago de Chile: Pontificia Universidad Católica de Chile.

Frosini, T. (2002). Subsidiariedad y Constitución. Revista de Estudios Políticos, (115), 7-26.

Generadoras de Chile. (2018). Boletín del mercado eléctrico: sector generación. Recuperado de http:// generadoras.cl/media/page-files/507/boletin-mercado-electrico-sector-generacion-junio-2018.pdf 
Guillén, J. (2010). Derecho de la competencia y regulación en el mercado eléctrico: el problema de las restricciones técnicas. En L. Cases (Dir.), Anuario de la Competencia 2009 (pp. 295-332). Barcelona: Servei de Publicacions de la Universitat Autònoma de Barcelona.

Jefatura del Estado. España. (1997). Ley 54/1997 de noviembre 27 del Sector Eléctrico. Boletín Oficial del Estado n. ${ }^{\circ} 285$ de noviembre 28 de 1997.

Jefatura del Estado. España. (2007). Ley 17/2007 de julio 4 por la que se modifica la Ley 54/1997, de 27 de noviembre, del Sector Eléctrico, para adaptarla a lo dispuesto en la Directiva 2003/54/CE, del Parlamento Europeo y del Consejo, de 26 de junio de 2003, sobre normas comunes para el mercado interior de la electricidad. Boletín Oficial del Estado n. ${ }^{\circ} 160$ de julio 5 de 2007.

Jefatura del Estado. España. (2013). Ley 24/2013 de diciembre 26 del Sector Eléctrico. Boletín Oficial del Estado n. 310 de diciembre 27 de 2013.

Joskow, P. (2005). Vertical integration. En C. Ménard y M. Shirley (Eds.), Handbook of new institutional economics (pp. 319-347). Berlín: Springer.

Mankiw, G. (2002). Principios de economía (Trads. E. Rabasco y L. Toharía). Madrid: McGraw.

Millán, J. (2013). Regulación económica, competencia e incentivos. En A. Ruiz (Dir.), Fundamentos de regulación y competencia. El diálogo entre derecho y economía para el análisis de las políticas públicas (pp. 113-162). Madrid: Iustel.

Ministerio de Minería. Chile. (1982). Decreto con Fuerza de Ley n. 1 de junio 22 que aprueba modificaciones al D.F.L. n. 4 de 1959, Ley General de Servicios Eléctricos, en materia de energía eléctrica. Diario Oficial de septiembre 13 de 1982.

Ministerio de Economía, Fomento y Reconstrucción. Chile. (2006). Decreto con Fuerza de Ley n. 4/20.018 de mayo 12 que fija texto refundido, coordinado y sistematizado del Decreto con Fuerza de Ley n. ${ }^{\circ}$ 1, de minería, de 1982, Ley General de Servicios Eléctricos, en materia de energía eléctrica. Diario Oficial de febrero 5 de 2007.

Ministerio de Economía, Fomento y Reconstrucción. Chile. (2004). Ley n. 19.940 de marzo 12 que regula sistemas de transporte de energía eléctrica, establece un nuevo régimen de tarifas para sistemas eléctricos medianos e introduce las adecuaciones que indica a la Ley General de Servicios Eléctricos. Diario Oficial de marzo 13 de 2004.

Ministerio de Energía. Chile. (2016). Ley n. 20.936 de febrero 9 que establece un nuevo sistema de transmisión eléctrica y crea un organismo coordinador independiente del sistema eléctrico nacional. Diario Oficial de julio 20 de 2016.

Montero, J. (2013). Regulación económica y derecho a la competencia: dos instrumentos complementarios de intervención pública para los mercados de interés general. En A. Ruiz (Dir.), Fundamentos de regulación y competencia. El diálogo entre derecho y economía para el análisis de las políticas públicas (pp. 81-111). Madrid: Iustel.

Muñoz, S. (2009). Fundamentos e instrumentos jurídicos de la regulación económica. En S. Muñoz y J. Esteve (Dirs.), Derecho de la regulación económica (Vol. 1) (pp. 15-244). Madrid: Iustel. 
Nebreda, J. (2008). Separación de actividades en el sector eléctrico. Serie Unión Europea y Relaciones Internacionales, (26), pp. 1-17.

Olivares, A. (2014). Libre mercado y regulación: la experiencia en el sector eléctrico español. Revista Chilena de Derecho, 41(1), 205-228.

Pajuelo, B. (2009). Transporte de energía eléctrica. En S. Muñoz y J. Esteve (Dirs.), Derecho de la regulación económica (Vol. 3) (pp. 535-578). Madrid: Iustel.

Parlamento Europeo y Consejo de la Unión Europea. (1996). Directiva 96/92/CE de diciembre 19 sobre normas comunes para el mercado interior de la electricidad. Diario Oficial de la Unión Europea n. ${ }^{\circ}$ L 027 de enero 30 de 1997.

Parlamento Europeo y Consejo de la Unión Europea. (2003). Directiva 2003/54/CE sobre normas comunes para el mercado interior de la electricidad y por la que se deroga la Directiva 96/92/ CE. Diario Oficial de la Unión Europea n. ․ L 176 de julio 15 de 2003.

Parlamento Europeo y Consejo de la Unión Europea. (2009). Directiva 2009/72/CE sobre normas comunes para el mercado interior de la electricidad y por la que se deroga la Directiva 2003/54/ CE. Diario Oficial de la Unión Europea n. ․ 211 de agosto 14 de 2009.

Pollitt, M. (2004). Electricity reform in Chile: lessons for developing countries. Journal of Network Industries, 5(3-4), 221-262.

Riesco, R. y Vial, V. (2015). La regulación de la integración vertical en el mercado eléctrico: una revisión al artículo $7^{\circ}$ de la Ley General de Servicios Eléctricos desde una perspectiva regulatoria y de libre competencia. Actas de Derecho de Energía, (5), 43-57.

Ruiz, H. (2014). Subsidios cruzados. La Prensa. Recuperado de https://www.laprensa.com.ni/2014/07/12/ economia/202872-subsidios-cruzados-3

Salinas, E. (2009). La actividad del comercializador. El paso de la tarifa regulada al suministro de último recurso. La Oficina de Cambios de Suministrador. En S. Muñoz y J. Esteve (Dirs.), Derecho de la regulación económica (Vol. 3) (pp. 765-810). Madrid: Iustel.

Sánchez, J. (2007). La separación de actividades en la Ley 17/2007, por la que se modifica la Ley 54/1997, de 27 de diciembre, del sector eléctrico, para adaptarla a la Directiva 2003/54/CE del Parlamento Europeo y del Consejo, de 26 de junio de 2003, sobre normas comunes para el mercado interior de la electricidad. En V. López-Ibor (Coord.), Comentarios a la Ley de reforma del sector eléctrico: Ley 17 de 2007 (pp. 56-125). Navarra: Civitas.

Schnake, E. (2015). Régimen de acceso abierto: condiciones de implementación y sus efectos. Actas de Derecho de Energía, (5), 59-73.

Sepúlveda, E. (2010). Sistema y mercados eléctricos. Santiago de Chile: Legal Publishing.

Serra, P. (2011). Competencia en los sectores eléctricos, las experiencias de Chile y el Reino Unido. En Tribunal de la Libre Competencia (Ed.), La competencia en el Chile del bicentenario (pp. 431-457). Santiago de Chile: Thomson Reuters. 
Stiglitz, J. (2000). La economía del sector público. Barcelona: Antoni Bosch.

Tamayo, M. y Piñeros, J. (2007). Formas de integración de las empresas. Ecos de Economía, (24), 27-45.

Vergara, A. (2004). Derecho eléctrico. Santiago de Chile: Editorial Jurídica.

Ybar, M. (2016). Comprendiendo el abuso de posición de dominio: revisión de la doctrina y jurisprudencia nacional y comparada sobre las principales conductas exclusorias. Revista de Derecho Económico, (76), 11-60. 